\title{
Effects of the disconnected flavor singlet corrections on the hyperfine splitting in charmonium
}

\section{DeTar and L. Levkova*}

University of Utah, Salt Lake City, UT 84112, USA

E-mail: detar@physics.utah.edu, ludmila@physics.utah.edu

\section{Fermilab Lattice and MILC Collaborations}

Experimentally the charmonium hyperfine splitting is $M_{J / \psi}-M_{\eta_{c}}=117 \mathrm{MeV}$ and current lattice results are generally below this value. The difference could be due to the effects of the disconnected flavor singlet diagrams which have not been included in these calculations. Previous attempts to determine the disconnected flavor singlet corrections have led just to rough estimates in the range of $\pm 20 \mathrm{MeV}$. We present preliminary results for these corrections calculated on fine $(a \approx 0.09 \mathrm{fm})$ Asqtad 2+1 flavor lattices provided by the MILC Collaboration.

The XXV International Symposium on Lattice Field Theory

July 30 - August 42007

Regensburg, Germany

\footnotetext{
* Speaker.
} 


\section{Introduction}

The lattice calculation of the hyperfine splitting in charmonium is currently still showing a discrepancy with the experimental value of $117 \mathrm{MeV}$. The discrepancy is large (30-40\%) in the quenched case [1,2]. It is reduced to around $10 \%$ in dynamical studies with improved actions [3, 4], but its origin is still uncertain. A possible explanation could be that even the current state-of-theart lattice action formulations do not reproduce the quark dynamics within the charmonium states sufficiently accurately for the purpose of this calculation. Another possibility which could account for the discrepancy (or at least part of it) is the neglected contribution of the disconnected diagrams in the lattice computations. These diagrams could contribute to the masses of both the vector $J / \psi$ and the pseudoscalar $\eta_{c}$, and thus affect the hyperfine splitting $M_{J / \psi}-M_{\eta_{c}}$. Perturbatively, the contribution of these diagrams in charmonium is expected to be small due to the OZI suppression, especially for the vector state. However, non-perturbative effects, such as the $U_{A}(1)$ anomaly [5] and glueball mixing, might enhance it enough so that it becomes a non-negligible fraction of the hyperfine splitting.

In this work, we present our preliminary results of the effort to test this possibility and determine the contributions of the disconnected diagrams. Previous calculations [6,7] using two-flavor gauge ensembles very roughly estimate the contribution to be within $\pm 20 \mathrm{MeV}$. They both confirm that there are significant difficulties in obtaining a signal for the disconnected diagrams due to noise, especially for heavy quarks. Our calculations are performed on an ensemble of 505, 2+1 flavor MILC lattices [8], generated with the improved Asqtad action [9]. For this ensemble the ratio of the masses of the light and heavy quarks is $m_{u d} / m_{s}=0.1$. The charm quarks are simulated using the Fermilab interpretation of the clover action with $\kappa_{c}=0.127$ tuned to the physical charm quark mass. The disconnected diagrams are calculated stochasticly with 12 spin and color diluted sources.

We employ several methods for improving our calculation in comparison with previous works. First, our lattice volume is $40^{3} \times 96$, which is much larger than in $[6,7]$ and our lattice scale, $a \approx 0.09 \mathrm{fm}$, is finer. In addition to these improvements we use the unbiased subtraction technique [10] in the stochastic estimators. The success of this technique depends on the fast convergence of the hopping parameter expansion used in the subtraction. Considering that $\kappa_{c}$ is small for the charm quark, we use the terms of the expansion only up to third order in $\kappa_{c}$, which reduces the standard deviation of the disconnected correlator by about a factor of 4 . The last and most important improvement is that we study the point-to-point (PP) disconnected correlators instead of the traditional time-slice-to-time-slice (TS) ones. As a result, the standard deviation of the signal is reduced about three orders of magnitude.

\section{Lattice formulation}

The disconnected part of the flavor singlet TS correlator is calculated as:

$$
D(t)=c_{\Gamma}\left\langle L(0) L^{*}(t)\right\rangle, \quad \text { where } \quad L(t)=\operatorname{Tr}\left(\Gamma M^{-1}\right)
$$

and the trace is over the Dirac, color and space indices. For the vector we have $\Gamma=\gamma_{\mu}, c_{\Gamma}=1$ and for the pseudoscalar $\Gamma=\gamma_{5}, c_{\Gamma}=-1$. To determine the effect of the disconnected diagram on the 
masses of the charmonium states, previous works $[6,7]$ explore the ratio of the disconnected to the connected TS correlators at zero momentum:

$$
\frac{D(t)}{C(t)}=\frac{F(t)}{C(t)}-1=\frac{A_{f}}{A_{c}} e^{\left(m_{c}-m_{f}\right) t}-1
$$

In the above $F(t)=D(t)+C(t)$ is the full propagator corresponding to a state with "full" mass $m_{f}$. The mass $m_{c}$ extracted from the connected propagator is the mass which is usually studied in lattice simulations. The constants $A_{f}$ and $A_{c}$ are the full and connected propagator amplitudes, respectively. Considering that the available lattices are quenched with respect to the charm quark, an appropriate fitting form for the ratio data at zero momentum would be

$$
\frac{D(t)}{C(t)}=\left(m_{c}-m_{f}\right) t+\frac{m_{c}-m_{f}}{m_{c}}
$$

The differences $m_{c}-m_{f}$ should be calculated for both the vector and the pseudoscalar and then subtracted from the mass of each in order to determine the effects of the disconnected diagrams on the hyperfine splitting.

In this work however, we calculate the PP disconnected correlators, and the above analysis has to be modified accordingly. The disconnected PP propagator as a function of the Euclidean distance on the lattice $r$ is defined as:

$$
D(r)=\frac{c_{\Gamma}}{N_{r}} \sum_{r=\left|x-x^{\prime}\right|}\left\langle L(x) L^{*}\left(x^{\prime}\right)\right\rangle,
$$

where the sum is over all pairs of lattice points at this distance, $N_{r}$ is the number of these pairs and there is trace only over spins and colors in $L$. The connected PP correlator $C(r)$ is defined in a similar manner. It is known that in the continuum limit the asymptotic behavior of $C(r)$ is:

$$
C(r) \sim A \frac{e^{-m_{c} r}}{r^{\frac{3}{2}}}
$$

and consequently the behavior of $D(r)$ in this case can be deduced as follows:

$$
D(r) \sim-\frac{d}{d m_{c}^{2}} C(r) \sim B \frac{e^{-m_{c} r}}{r^{\frac{1}{2}}} .
$$

Thus their ratio is

$$
\frac{D(r)}{C(r)} \approx \frac{B}{A} r
$$

where we interpret the amplitude ratio as:

$$
\frac{B}{A}=m_{c}-m_{f}
$$

The behavior of $D(r)$ and $C(r)$ on the lattice will be affected by discretization artifacts; still, for large $r$ and fine lattices we assume that the above behavior is a good approximation. 


\section{Results for $D(r)$}

From the previous studies it is known that the charmonium correlator signal disappears very quickly around $t=2-3$. We work with the PP disconnected propagator, since this way we benefit from both the additional data at non-integer distances and the much improved statistics. The correlator $D(r)$ has from one to three orders of magnitude smaller relative errors than the TS disconnected propagator in the region where we have a signal. Figure 1 illustrates this statement by comparing $D(r)$ and $D(t)$ for $\eta_{c}$ for two different ranges of $r$ and $t$. In the right panel of Fig. 1, the comparison is done on a shorter range in order to emphasize the fact that we do have a clear signal for $D(r)$ in the range where the $D(t)$ signal is completely obscured by the noise. The result
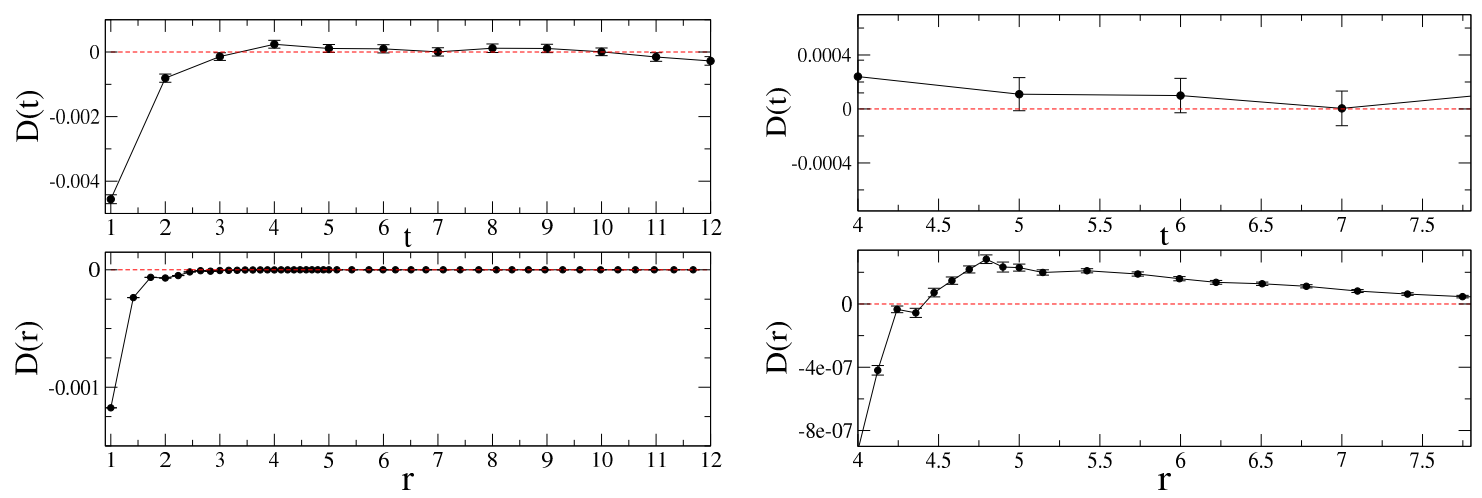

Figure 1: Comparison of $D(t)$ and $D(r)$ for $\eta_{c}$ for two different ranges of $t$ and $r$.

that the $D(r)$ signal is so much better than the one for $D(t)$ can be explained by the fact that in the calculation of $D(t)$ there are a great number of contributions from points, which although not far from each other in the $t$ direction, are far in the $4 \mathrm{~d}$ Euclidean space. For the disconnected correlator, the noise increases strongly with the distance and such points contribute nothing to $D(t)$ but noise. This problem is avoided when working with $D(r)$ instead.

\section{The $D(r) / C(r)$ ratio for $\eta_{c}$}

Figure 2 shows our data for the ratio $D(r) / C(r)$. We make the following observations:

- The roughness of the data at short distance is a sign of rotational symmetry breaking and requires a nonasymptotic lattice fitting function.

- The ratio $D(r) / C(r)$ inherits the sign flip of $D(r)$. The sign flip is expected, since from the CP symmetry of the operator, the sign of $D(r)$ at $r=0$ must be opposite the sign of any singlepole contributions, and they dominate at large $r$. We believe this is the first observation of light state contributions to an operator constructed from charm quarks.

- In a separate analysis we find that excited states in the TS correlator $C(t)$ contribute more than $20 \%$ for $r<5$. The same is expected for $D(r)$. Indeed, if we assume a single heavy mode at short distance, to make the nonasymptotic form of $D(r) / C(r)$ resemble the data, we need a mass much heavier than the $\eta_{c}$, as indicated in the right panel of Fig. 2. The light 

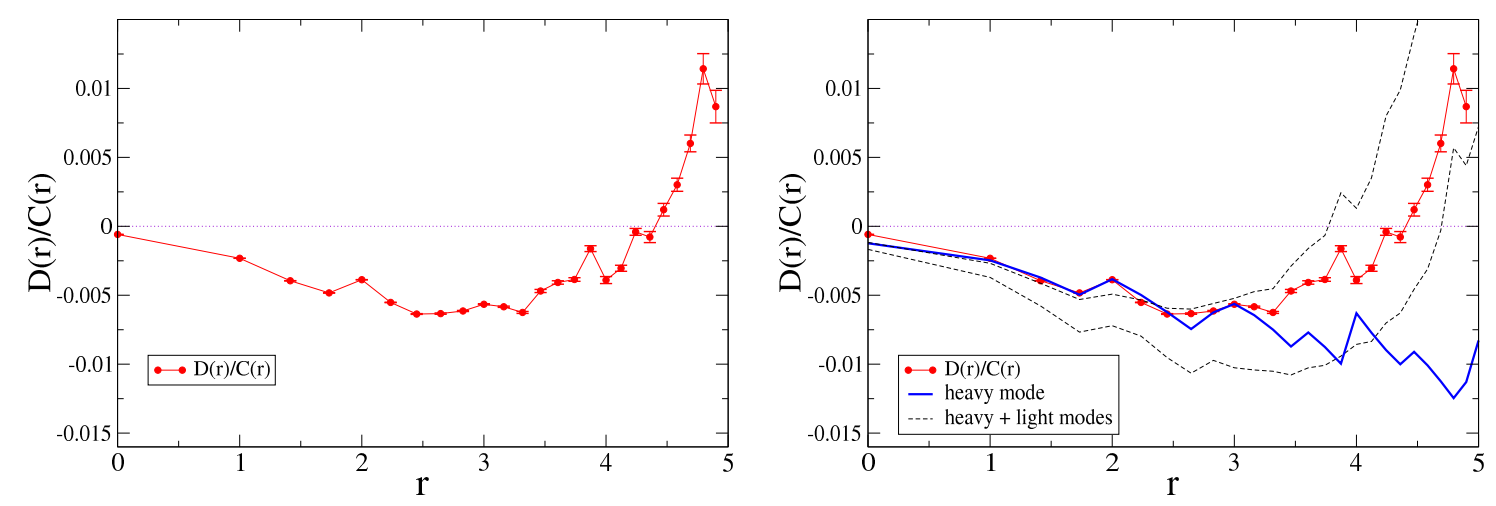

Figure 2: The lattice data for the ratio $D(r) / C(r)$ for $\eta_{c}$. In the right panel the actual data is compared with theoretical models with a heavy mode only or with a sum of heavy and light modes.

states in $D(r)$ are very likely dominant for $r>4$, but we estimate that the charmonium signal is still about $30 \%$ of the whole signal at $r=5$.

- The asymptotic expression Eq. (2.7) is not likely to be applicable anywhere. At large distance $D(r)$ is dominated by lighter hadronic modes (the $\eta$ and $\eta^{\prime}$ mesons, glueballs, etc.). Thus to extract the ratio of ground state amplitudes we must resort to more elaborate models.

- In the right panel of Fig. 2 we present two more exploratory approximations for $D(r) / C(r)$ that include one heavy and one light mode, just to show that the behavior of the ratio data can be approximated in this manner.

\section{Extracting the ground state $\eta_{c}$ signal from $D(r)$}

Following the discussion in the previous section, at present we are led to explore a simplified model for the intermediate range $r \in[5,11]$ that includes a ground and excited $\eta_{c}$ state and a light state. The light state represents a number of possible states. We work separately with $D(r)$ and $C(r)$, extract the amplitudes of the $\eta_{c}$ contributions, and finally use Eq (2.8) to get an approximate mass shift.

For the connected correlator we have only a connected- $\eta_{c}$ ground state and one excited state. For the disconnected correlator our ansatz includes a light state as well. In momentum space it reads

$$
D\left(p^{2}\right)=\left[\frac{f\left(p^{2}\right)}{p^{2}+m_{c}^{2}}+\frac{g\left(p^{2}\right)}{p^{2}+m_{c}^{* 2}}\right]^{2} \frac{1}{p^{2}+m_{l}^{2}}
$$

where $m_{l}$ is a light state mass, $m_{c}^{*}$ is an excited connected $\eta_{c}$ mass, and $f\left(p^{2}\right)$ and $g\left(p^{2}\right)$ are real functions. We need the residue of the double pole at $p^{2}+m_{c}^{2}=0$ to determine the mass shift. In coordinate space the asymptotic form is then

$$
D^{f i t}(r)=\frac{B}{r^{\frac{1}{2}}}\left(e^{-m_{c} r}+e^{-m_{c}^{*} r}\right)+\frac{c B}{r^{\frac{3}{2}}}\left(e^{-m_{c} r}-e^{-m_{c}^{*} r}\right)+\frac{L}{r^{\frac{3}{2}}} e^{-m_{l} r} .
$$

The terms correspond, respectively, (Fig. 3) to double pole contributions from the ground and excited charmonium states, mixed ground-excited state contributions, and the light state contribution. 


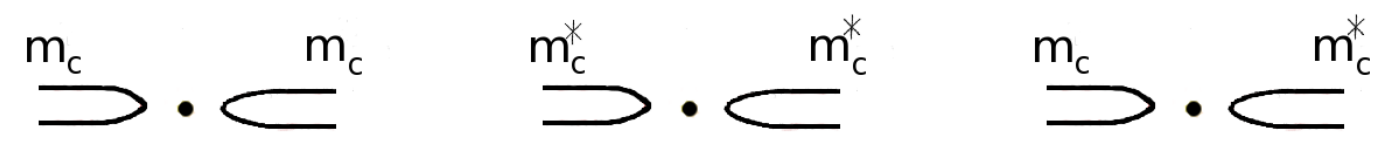

Figure 3: Charmonium disconnected diagrams included in the fitting form Eq. (5.2). The ground state diagram is to the left, the excited is in the middle and the "mixed" one is to the right.

For this preliminary analysis, we take the amplitudes $(B)$ of the ground and excited charmonium states to be equal. We do this in order to reduce the number of free parameters. Since the excited state contribution is just a small correction in the chosen fit range, this restriction on the amplitude is not of great importance. The coefficient $c$ is determined from the mass terms in the ansatz. We fix $c \approx 7$, but find that our results are not very sensitive to varying it over the range $c \in[2,14]$.

We adjust only $B$ and $L$. We fix the masses as follows: The light mass is taken to be the central value $m_{l}=0.43(1)$ from a single-exponential fit (as in Eq. (2.5)) in the range $r \in[7,12]$. The connected $\eta_{c}$ and $\eta_{c}^{*}$ masses, $m_{c}=1.1598(7)$ and $m_{c}^{*}=1.51(5)$, are known from fits to the connected TS propagator $C(t)$.

Before fitting we smoothed the data by averaging the signal in small bins in $r$, thus reducing the effects of the lattice artifacts and improving the continuum approximation. The resulting fit to $D(r)$ is shown in Fig. 4. The fit has $\chi^{2} / d f=24 / 19$. Our fit result favors the following range of

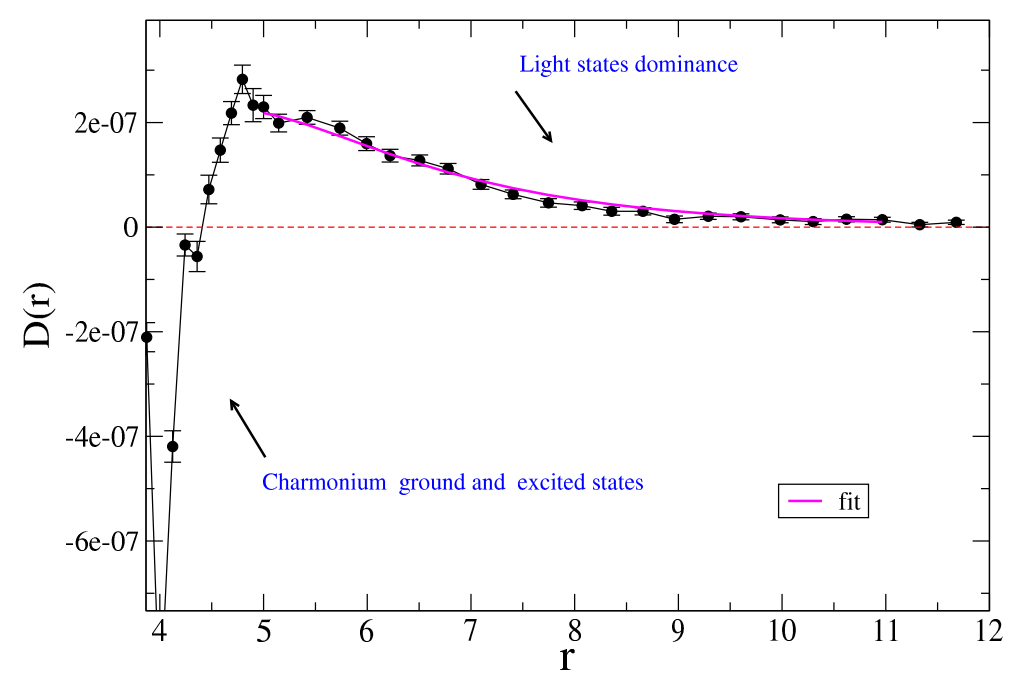

Figure 4: The disconnected PP correlator $D(r)$ and fit to it in the range of distances $r \in[5,11]$.

values for the ratio:

$$
\frac{B}{A}=m_{c}-m_{f} \in[-4,-1] \mathrm{MeV} .
$$

Thus the disconnected diagram contribution slightly increases the $\eta_{c}$ mass. 


\section{Summary and conclusions}

We have calculated the disconnected pseudoscalar propagator with dramatically reduced noise using unbiased subtraction methods and point-to-point correlator data. We fit the data to a simplified model and extracted the amplitudes of the ground state in the disconnected and connected propagators. We find that the disconnected diagram contribution increases the $\eta_{c}$ mass by $1-4 \mathrm{MeV}$.

The vector meson $J / \psi$ can be studied in a similar fashion. If the corresponding mass shift of the $J / \psi$ is negligible as expected [6,7], we would then conclude that the hyperfine splitting is reduced slightly.

This result is preliminary, however. Further work is needed to explore the sensitivity of this conclusion to the choice of the fitting model and to include effects of rotational symmetry breaking properly. Calculations at a smaller lattice spacing are needed to test for heavy-quark and hard-gluon discretization errors. A similar study of the $J / \psi$ could test the common expectation that its mass shift is negligible.

\section{Acknowledgments}

We are grateful to Peter Lepage for helpful comments. This work was supported by the US DOE and NSF. Computations were performed at CHPC (Utah) and FNAL.

\section{References}

[1] S. Choe et al. [QCD-TARO Collaboration], JHEP 08 (2003) 022 [ hep-lat / 0307004 ]

[2] H. D. Trottier, Phys. Rev. D55 (1997) 6844 [hep-lat/9611026] ; N. H. Shakespeare and H. D. Trottier, Phys. Rev. D58 (1998) 034502 [hep-lat/9802038] .

[3] S. Gottlieb et al., PoS(LAT2006) 175.

[4] E. Follana et al., Phys. Rev. D75 (2007) 054502 [hep-lat / 054502 ] .

[5] G. 't Hooft, Phys. Rev. Lett. 37 (1976); T. Schäfer and E. V. Shuryak, Rev. Mod. Phys. 70 (1998) 323.

[6] C. McNeile and C. Michael, Phys. Rev. D70 (2004) 034506 [ hep-lat / 0402012 ] .

[7] P. de Forcrand et al. [QCD-TARO Collaboration], JHEP 0408 (2004) 004 [ hep-lat / 0404016 ].

[8] C. Bernard et al. [MILC Collaboration], PoS LAT2005 (2006) 025 [hep-lat/ 0509137 ] .

[9] K. Orginos and D. Toussaint, Phys. Rev. D59 (1999) 014501; Nucl. Phys. Proc. Suppl. 73 (1999) 909; G. P. Lepage, Nucl. Phys. Proc. Suppl. 60A (1998) 267; Phys. Rev. D59 (1999) 074502.

[10] C. Thron et al., Phys. Rev. D57, 1642 (1998). 